\title{
НЕОПРЕДЕЛЕННОСТИ НЕСТОХАСТИЧЕСКОГО ТИПА ПРИ ФОРМИРОВАНИИ ОБЛИКА КОЛЕСНОЙ ТЕХНИКИ
}

\section{NON-STOCHASTIC UNCERTAINTIES IN THE FORMATION OF THE APPEARANCE OF WHEELED VEHICLES}

\section{E. Mikhailova \\ A. Amosov}

Summary. In this paper, the team of authors set a task before starting to form the appearance of aggregates, to consider the traditional approach to solving the problem and note the features of this process in relation to the considered class of machines.

The process of designing any complex technical objects, and transport units in particular, usually has a specific character. First of all, this is due to such contradictory requirements as increasing the load capacity while reducing the total weight and size of the machines. The increase in load capacity, in turn, leads to the need to improve the strength characteristics of both the chassis and the lifting mechanism of the machine, in accordance with the stated performance characteristics.

The problem of transporting goods within a certain area requires significant expenditures for the construction of new infrastructure and makes it impossible to use the existing one due to the increase in massdimensional characteristics. It is caused by, among other things, the geometric characteristics of the transported goods and requires solving a number of scientific and technical problems.

Keywords: unit, special transport, design, layout, creation, stages of creation, algorithm, appearance, cargo transportation, expert assessment.

\section{Ввемение}

D настоящее время в мире колесных и гусеничных агрегатов наблюдается устойчивая тенденция к разделению на машины низкой, средней и высокой грузоподъемности. В первую очередь это связано с существенным различием выполняемых такими машинами задач. Например, для погрузки и перевозки, небольших по массе грузов (до 3 тонн) как правило, используются мобильный кран - манипулятор с грузовой платформой, для эвакуации и перевозки автомобилей - специально предназначенный для этих целей ав-
Михайлова Екатерина Вячеславна

Ассистент, Московский авиационный институт (национальный исследовательский университет) evmihailova@inbox.ru

Амосов Алексей Германович Ассистент, Московский авиационный институт (национальный исследовательский университет) lamosov@yandex.ru

Аннотация. В данной работе коллектив авторов поставил перед собой задачу перед тем, как приступать к формированию облика агрегатов, рассмотреть традиционный подход к решению задачи и отметить особенности этого процесса применительно к рассматриваемому классу машин.

Процесс проектирования любых сложных технических объектов, и транспортных агрегатов в особенности, как правило, имеет специфический характер. В первую очередь это связано с такими противоречивыми требованиями, как увеличение грузоподъемности при снижении полной массы и размеров машин. Рост грузоподъемности в свою очередь приводит к необходимости повышать прочностные характеристики, как шасси, так и подъемного механизма машины, в соответствии с заявленными эксплуатационными характеристиками.

Проблема транспортировки грузов в пределах определенного района требует значительных расходов на возведение новой инфраструктуры и делает невозможным использование имеющейся из-за увеличения массово-габаритных характеристик. Обуславливается она, в том числе геометрическими характеристиками перевозимых грузов и требует решения целого ряда научно-технических задач.

Ключевые слова: агрегат, специальный транспорт, проектирование, компоновка, создание, этапы создания, алгоритм, облик, перевозка груза, экспертная оценка.

тоэвакуатор, а для подъема на большую высоту тяжелых строительных конструкций - мощные и высокопроизводительные краны с телескопической стрелой.

Многолетний опыт эксплуатации такой техники, как в нашей стране, так и за рубежом однозначно показывает, что невозможно создать универсальную машину, которая позволяла бы решать весь спектр операций. Повышение грузоподъемности таких машин, как наиболее очевидный путь к повышению их универсальности, неизбежно ведет к потере ими возможности выполнять целый ряд задач, например, погрузочные работы в ус- 

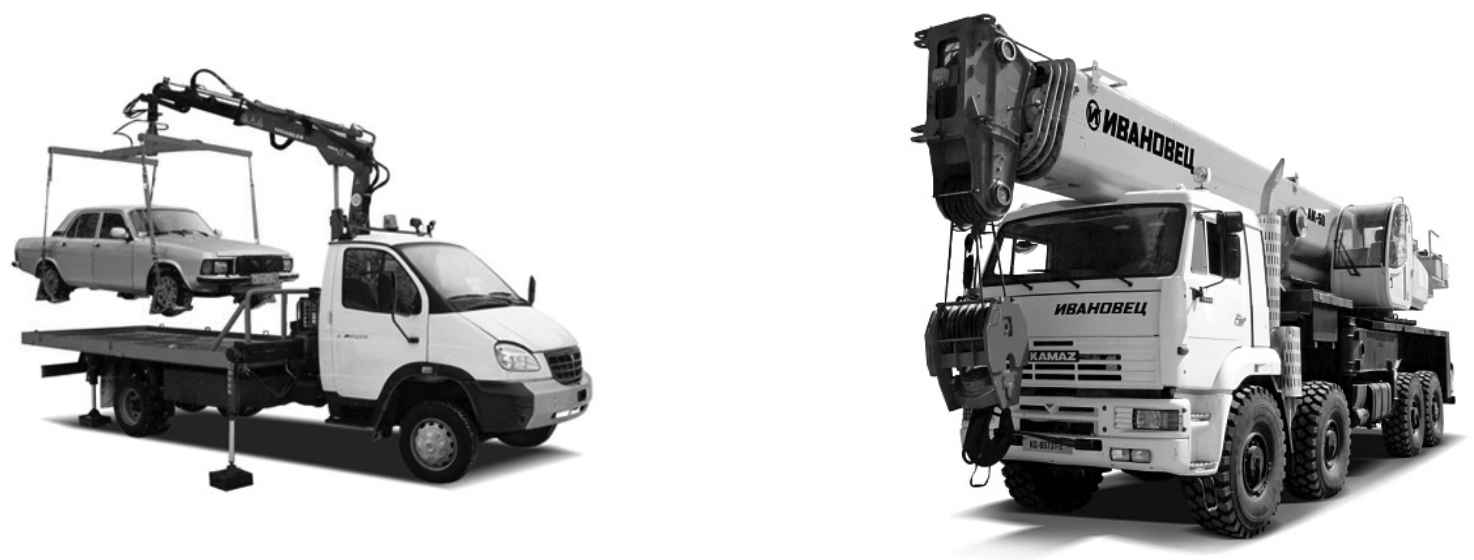

Рис. 1. Автокран и автоэвакуатор

ловиях ограниченного пространства, или совмещение операций погрузки и транспортировки грузов (рис. 1). В таких ситуациях на помощь машинам с большой грузоподъемностью приходят лёгкие автопогрузчики с высокой манёвренностью, малыми габаритами и относительно низкой стоимостью.

\section{Мето ология}

Под обликом в данном диссертационном исследовании понимается определение значений основных конструктивных параметров, которые однозначно определяют форму, размеры и эксплуатационные характеристики таких агрегатов, соответствующие этапу их предварительного проектирования.

На данном этапе необходимо, в первую очередь, корректно согласовать требования внешнего и возможно внутреннего проектирования. Удовлетворение этих требований станет результатом некоторого компромиссного решения отдельных задач проектирования. Далее требуется определить содержание задач, решаемых на каждом уровне, и установить связи между ними.

Блок-схема алгоритма процесса формирования технического облика состоит из этапов, показанных на рис. 2 [10]. Рассмотрим эти этапы подробно.

На первом уровне данного алгоритма определяются цели и задачи проектирования, требования, сформулированные в общем виде в техническом задании, переводятся в схемные решения. Целью является определение оптимальных компоновки и их технических характеристик.

Например, требования по грузоподъемности телескопической стрелы может быть выполнены за счет применения альтернативных вариантов профиля ее секций, которые способны обеспечить требуемую жесткость всей конструкции в заданном диапазоне нагрузок.

На втором уровне проводится определение основных показателей, характеризующих технический уровень агрегатов в целом: грузоподъемности, соотношения полной массы к полезной нагрузке, мощности силовой установки, вылета стрелы, наличия и вместимости грузовой платформы и т.д. Далее выбирается концепция проектирования, исходя из класса агрегата малой, средней или большой грузоподъемности (Рис. 3). Также производится ранжирование этих показателей по степени их влияния на технический облик, с целью определения уровней значимости всех определяющих параметров.

Затем, в рамках выбранной концепции, определяются такие параметры, как состав и варианты перевозимого груза и требования по его размещению. Далее, в зависимости от заданной массы полезного груза, оборудования и эксплуатационных материалов в первом приближении определяются расчётным путём основные массовые характеристики агрегата. Это первый этап весового проектирования агрегата, он характеризуется определением полной массы, выявлением групп элементов, определяющие параметры которых известны, а также контролем над соотношением этих параметров для отдельных элементов агрегата.

Основные трудности, возникающие на этом этапе разработки, заключаются в следующем:

- нет четкой методики формирования номенклатуры критериев, которые наиболее полно характеризуют технический уровень разрабатываемых агрегатов;

- не обозначены четкие границы, в рамках которых должны находиться значения наиболее важных определяющих параметров; 


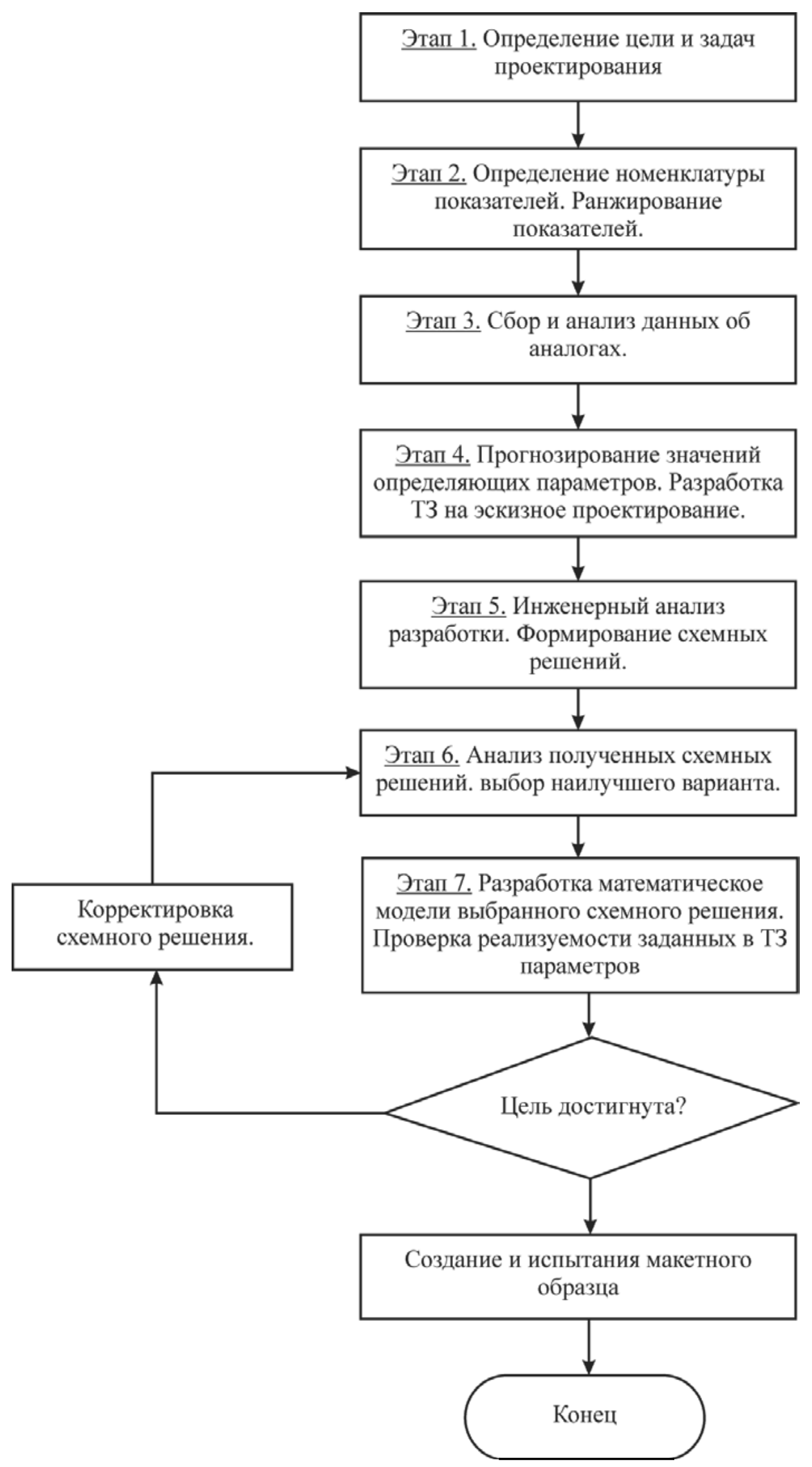

Рис. 2. Схема алгоритма формирования облика агрегатов 

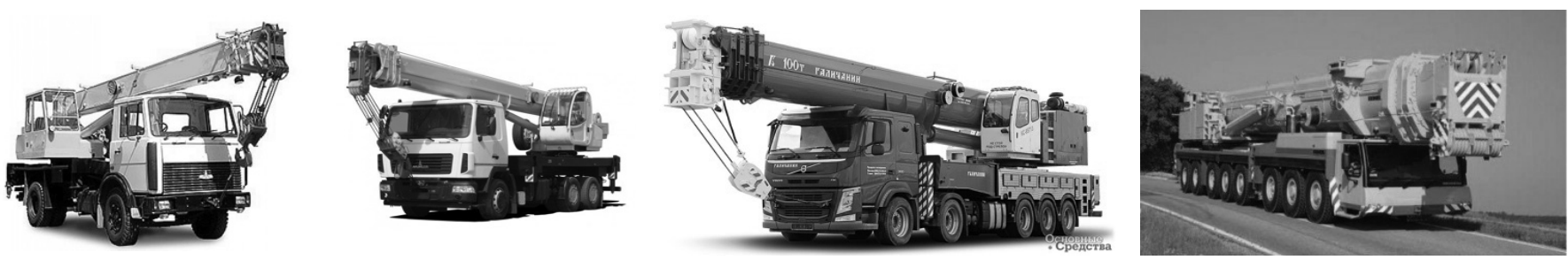

Рис. 3. Автокраны разной грузоподъемности $(25,32,100,500$ тонн)

- отсутствуют методики ранжирования определяющих параметров по степени их важности, поэтому требуется использование экспертных оценок;

- достаточно ограниченный объем информации по проектируемым объектам, что не позволяет в полной мере использовать статистические методы предварительной обработки информации.

В реальной практике при разработке технического задания на какой - либо технический объект часто приходится сталкиваться со случаями, когда количественно обосновать отдельные показатели нет возможности, поэтому степень проявления таких свойств возможно оценить только качественно, например, «высокий уровень», «средний уровень», «низкий уровень». В таких случаях для ранжирования показателей применяется экспертная оценка на основе сравнения рассматриваемых определяющих параметров [1].

Наибольшее распространение среди методов экспертной оценки таких объектов получил метод парных сравнений Саати [2]. Особенность его использования заключается в необходимости нахождения собственного вектора матрицы парных сравнений, которая задается с помощью специальных таблиц параметров или шкал.

Третий этап посвящен сбору и анализу данных об аналогах и формированию базы знаний о проектируемом классе агрегатов. Группа аналогов разрабатываемых объектов строится на основе глубокого анализа информации о лучших зарубежных и отечественных образцах таких объектов, разрабатываемых в течение 10 и более лет [3].

На этом этапе решаются следующие задачи:

- определение достигнутого на сегодняшний день технического уровня в проектировании;

- анализ тенденций изменения значений определяющих параметров.

Данный этап характеризуется следующими трудностями:

1. количество выявленных аналогов почти всегда достаточно ограничено;
2. численные значения требуемых значений определяющих параметров далеко не всегда известны.

В этой связи проводить статистический анализ численных значений полученных характеристик невозможно, тем более невозможно осуществлять прогноз изменения этих параметров на перспективу, пользуясь статистическими методами [4].

На четвертом этапе выполняется прогнозирование значений определяющих параметров на 15-20 летний срок, который проходит в среднем от начала разработки до списания (утилизации) первых серийно выпускаемых единиц наземной колесной техники [5]. Основной проблемой, возникающей на данном этапе заключается в том, что полученные на предыдущем этапе значения определяющих параметров не являются временным рядом в классическом понимании [6]. Поэтому никакие статистические и интеллектуальные методы прогнозирования значений определяющих параметров на перспективу неприменимы. С другой стороны, можно привлечь экспертов, которые могут оценить, каким должно быть значение определяющего параметра в «идеальном» случае. Однако мнение экспертов в определенной степени носит субъективный характер. Единственная возможность - построение прогнозов на основе баланса мнений экспертов и объективных (даже ели они малочисленные) данных об изменении значения данного параметра во временной перспективе методами нечеткой логики [7].

Итогом выполнения работ данного этапа является обоснование количественных значений основных определяющих параметров в техническом задании на эскизное проектирование.

Пятый этап - проведение инженерного анализа разработки, формирование схемных решений, удовлетворяющих полученным значениям определяющих параметров, выполнение эскизного проекта. При этом решаются следующие задачи [8].

1. Предварительное формирование облика машины, отвечающего требованиям технического за- 
дания. Происходит формирование допустимой области существования проекта, где находится допустимое решение, описываемое параметрами начального приближения.

2. Оптимизация проектных параметров по выбранному критерию с учетом ограничений, вытекающих из требований технического задания и действующих нормативных документов.

3. Параметрический анализ, направленный на оценку чувствительности проекта к изменению его альтернатив (вариантов схемных решений), параметров и ограничений.

4. Уточнение характеристик изделия и документирование результатов проектирования.

Следующий, шестой этап включает в себя работы по анализу полученных схемных решений по результатам эскизного проектирования и выбору оптимального варианта агрегата. Однако, в процессе принятия решения выполняется многокритериальный анализ в условиях большого количества неопределенностей, когда цели и ограничения заданы нечеткими множествами. Принятие решений рассматриваемых условий - альтернатива, которая одновременно удовлетворяет и нечетким ограничениям, и нечетким целям. Решение задачи выбора оптимального схемного решения может быть выполнено с помощью теории принятия решений в нечетких условиях на основе задачи Беллмана-Заде на основе многокритериального анализа вариантов при равновесных и неравновесных критериях [9]. Весовые значения критериев получают на основе парных сравнений [7].

Седьмой этап - разработка математических моделей создаваемого агрегата специального назначения. Математическое моделирование позволяет провести всестороннее комплексное исследование выбранного схемного решения до создания натурного образца, что позволяет сэкономить время и средства, отпущенные на разработку. Задача этапа состоит в подтверждении реализуемости заданных значений определяющих параметров.

Затем производится формирование компоновочной схемы, заключающегося в осуществлении взаимной пространственной увязки основных элементов объекта. Компоновка происходит на базе выбранной на данном этапе схемы, включающей определяющие параметры. В процессе компоновки определяются внешние и внутренние формы, компонуется полезная нагрузка, оборудование, снаряжение, размещаются агрегаты подъемного механизма и т.д.

Этот этап заканчивается расчётом массы и других характеристик агрегата во втором приближении и уточ- нением основных определяющих параметров. Оценка компоновки проводится по частным критериям эффективности, что позволяет исключить из рассмотрения заведомо неэффективные варианты. Большинство из частных критериев являются взаимосвязанными, что не позволяет их использовать при оценке проектов, поскольку традиционный в этом случае подход, заключающийся в ведении весовых коэффициентов, существенно снижает объективность оценок.

Далее проводится окончательный расчёт определяющих параметров агрегатов. По результатам компоновки уже известны основные размеры, формы и основные характеристики.

Исходя из полученных величин, принимается решение о ходе дальнейшего проектирования.

Заканчиваются работы на этом этапе расчетом характеристик функционирования. Они характеризуют агрегат как сформированный объект.

Восьмой этап - проверка условия «Цель достигнута?». Если «Да», то можно переходить к процессу создания и испытаний. Если «Нет» - необходимо вернуться к анализу схемных решений (шестому этапу).

В случае удовлетворения всех требовании внешнего проектирования и отсутствия противоречий на этапах внутреннего проектирования процесс формирования облика агрегата заканчивается техническим предложением, т.е. выпуском чертежей общего вида, компоновки, трёхмерной каркасно-кинематической модели, а так же выдачей характеристик и результатов по частным критериям эффективности всех уровней.

\section{Обсу>кАения}

Налицо противоречие, которое заключается в том, что с одной стороны, для выполнения большинства погрузочных и транспортировочных работ требуются машины средней и большой грузоподъемности, с другой стороны, такие машины и так имеют значительную полную массу, и ее дальнейшее повышение неизбежно ведет к потере ими возможности выполнять целый ряд задач, особенно в условиях бездорожья и ограниченного пространства. Поэтому сложившаяся практика деления таких машин на категории по грузоподъемности вполне оправдана.

При формировании технического облика любого сложного технического объекта возникает еще одна существенная проблема - проблема неопределенности целей и неполноты информации. Причем традиционный путь учета факторов неопределенности на основе веро- 
ятностного и статистического моделирования зачастую оказывается неадекватным решаемым задачам и может привести к неверным результатам, так как функционирование сложных организационно-технических систем на практике характеризуется неопределенностью «нестохастического» типа вследствие:

- неполноты или отсутствия знаний о поведении отдельных входящих в систему элементов и подсистем, а также взаимосвязей между ними;

- невозможности или ограниченной возможности экспериментального исследования процессов, не позволяющей получить достаточную статистическую информацию о наиболее важных характеристиках системы.

Для моделирования, проектирования и анализа таких организационно-технических систем получили распространение экспертные системы, оперирующие экспертными оценками, основанными на опыте и знаниях эксперта (или группы экспертов).

\section{Выво $\triangle \mathrm{b}$}

Анализ процедур проектирования показывает, что они, сохраняя общий состав, присущий подвижным объектам, использующим колесный ход, практически неизменным, должны иметь развитие, отражающее присущую им специфику, либо быть разработаны заново.

При компоновке оборудования агрегатов задача компоновки его подъемного механизма должна быть выделена в отдельную подзадачу, а решения, принятые в её рамках, во многом определяют эффективность агрегата в целом.

Таким образом, в рамках проведенного поэтапного анализа процесса формирования технического облика агрегатов для самых сложных условий эксплуатации выявлены основные особенности каждого этапа и предложена номенклатура основных параметров, определяющих технический облик агрегатов.

\section{ЛИТЕРАТУРА}

1. Плиев И. А. Автомобили многоцелевого назначения. Формирование технического облика АМН в составе семейств: Монография.—- М.: МГИУ, 2011.— 262 c.

2. Саати Т. Принятие решений. Метод анализа иерархий. - М.: Радио и связь, 1993.— 278 с.

3. Белоусов Б.Н., Попов С. Д. Колесные транспортные средства особо большой грузоподъемности. Конструкция. Теория. Расчет. / Под общ. ред. Б. Н. Белоусова.—М.: Изд-во МГТУ им. Н. Э. Баумана, 2006. - 728 с

4. Интеллектуальный анализ временных рядов: учебное пособие // Н. Г. Ярушкина, Т. В. Афанасьева, И. Г. Перфильева. --Ульяновск: УлГтУ, 2010.—- 320 c.

5. ГОСТ 2.116-84. Карта технического уровня и качества продукции. Межгосударственный стандарт.-М.: Госстандарт СССР, 1984. - 16 с.

6. Анализ временных рядов и прогнозирование: учебник / В. Н. Афанасьев, М. М. Юзбашев.— М.: Финансы и статистика, 2001. - 228 с.

7. Ротштейн А.П., Штовба С. Д. Нечеткий многокритериальный анализ вариантов с применением парных сравнений // Известия РАН. Теория и системы управления.—2001.- No3.-С. 150-154.

8. Дьяков И. Ф. Оптимальное проектирование грузовых автомобилей.—Саратов: Издательство Саратовского университета, 1989. — 128 с.

9. BellmanR.E., Zadeh L. A. Decision-Makingin Fuzzy Environment // Management Science. — vol. 17. — 1970.— No4.—P. 141-160.

10. Калимулин М. Р. Анализ процесса формирования технического облика особо лёгких высокоподвижных колёсных транспортных средств для горных условий эксплуатации // Наука и образование.— 2012.— № 11.—c. 125

( ) Михайлова Екатерина Вячеславна ( evmihailova@inbox.ru ), Амосов Алексей Германович ( lamosov@yandex.ru ).

Журнал «Современная наука: актуальные проблемы теории и практики» 